\section{Eco.Mat}

Cúcuta-Colombia

Vol. 6

No. 1

Enero-Diciembre 2015 ISSN 1794-8231

ISSN 2462 - $8794 \mathrm{Web}$

PP: $116-124$

\title{
La violencia en la escuela
}

\author{
Violence at school
}

Jesús Villamizar-Ibarra ${ }^{1}$

Forma de citar: J. Villamizar. La violencia en la escuela. Eco.Mat. 2015; 6(1): 116-124.

Recibido:

Abril 20 de 2015

Aceptado:

Junio 10 de 2015
116

Abogado (Universidad Libre),Contador Público (U.F.P.S), Licenciado en Ciencias de la Educación-Ciencias Empresariales (U.F.P.S), Especialista en Docencia Universitaria (Universidad Santo Tomas), Especialista en Derecho Empresarial ( Universidad Autónoma de Bucaramanga), Magisters en Practicas Pedagógicas (U.F.P.S.), Docente Adscrito al Departamento Contable y Financiero. Correo: jesusvillamizar@ufps.edu.co.

\section{RESUMEN}

El problema de violencia en las aulas es un tema muy generalizado en los ambientes de enseñanza. Las situaciones de acoso en el ámbito escolar incluyen tanto al alumnado como a los propios docentes, con quienes se debe trabajar para comprender y reducir el problema que tanto afecta la convivencia pacífica. El objetivo del presente artículo de revisión fue analizar elementos estructurales en materia de violencia en la escuela, teniendo en cuenta los elementos culturales y educativos del mundo actual, que involucran diversos aspectos como la pedagogía violenta relacionada con la burocratización de la organización educativa y el gremialismo socio económico. Se aplicó el método de selección de información de carácter explicativo y analítico de investigación documental, según fuentes secundarias de información, con el objeto de familiarizar al investigador con el tema objeto del conocimiento de tipo cualitativo. El documento resalta los principios de participación, la corresponsabilidad, la autonomía, la diversidad y la integralidad, reconocimiento a los niños, niñas y adolescentes como sujetos de derechos y a la comunidad educativa como la responsable de formas para el ejercicio de los mismos, promulgados por el Sistema Nacional de Convivencia Escolar y recientemente por la Ley 1732 de Septiembre de 2014, por la cual se establece la cátedra de la paz en todas las instituciones educativas del país, con la finalidad de garantizar la creación y el fortalecimiento de una cultura de paz en Colombia.

Descriptores: violencia escolar, agresión, paz, competencias, tolerancia.

\begin{abstract}
The problem of violence in schools is a widespread issue in teaching environments. Situations of bullying in schools include both students and teachers themselves, with whom they must work to understand and reduce problem affecting both peaceful coexistence. The aim of this review article was to analyze structural elements on violence in school, taking into account cultural and educational elements of today's world, involving various aspects such as violent pedagogy related to the bureaucratization of the educational organization and social economic partner. The method of selection of explanatory information and analytical documentary research was applied as secondary sources of information, in order to familiarize the researcher with the subject matter of qualitative knowledge. The paper highlights the principles of participation, responsibility, autonomy, diversity and inclusiveness, recognizing children and adolescents as subjects of rights and the educational community as responsible for ways to exercise them, promulgated by the National System of School Coexistence and recently by Law 1732 of September 2014, by which the chair of peace is established in all educational institutions in the country, in order to ensure the establishment and strengthening a culture of peace in Colombia
\end{abstract}

Descriptors: school violence, aggression, peace, skills, tolerance. 


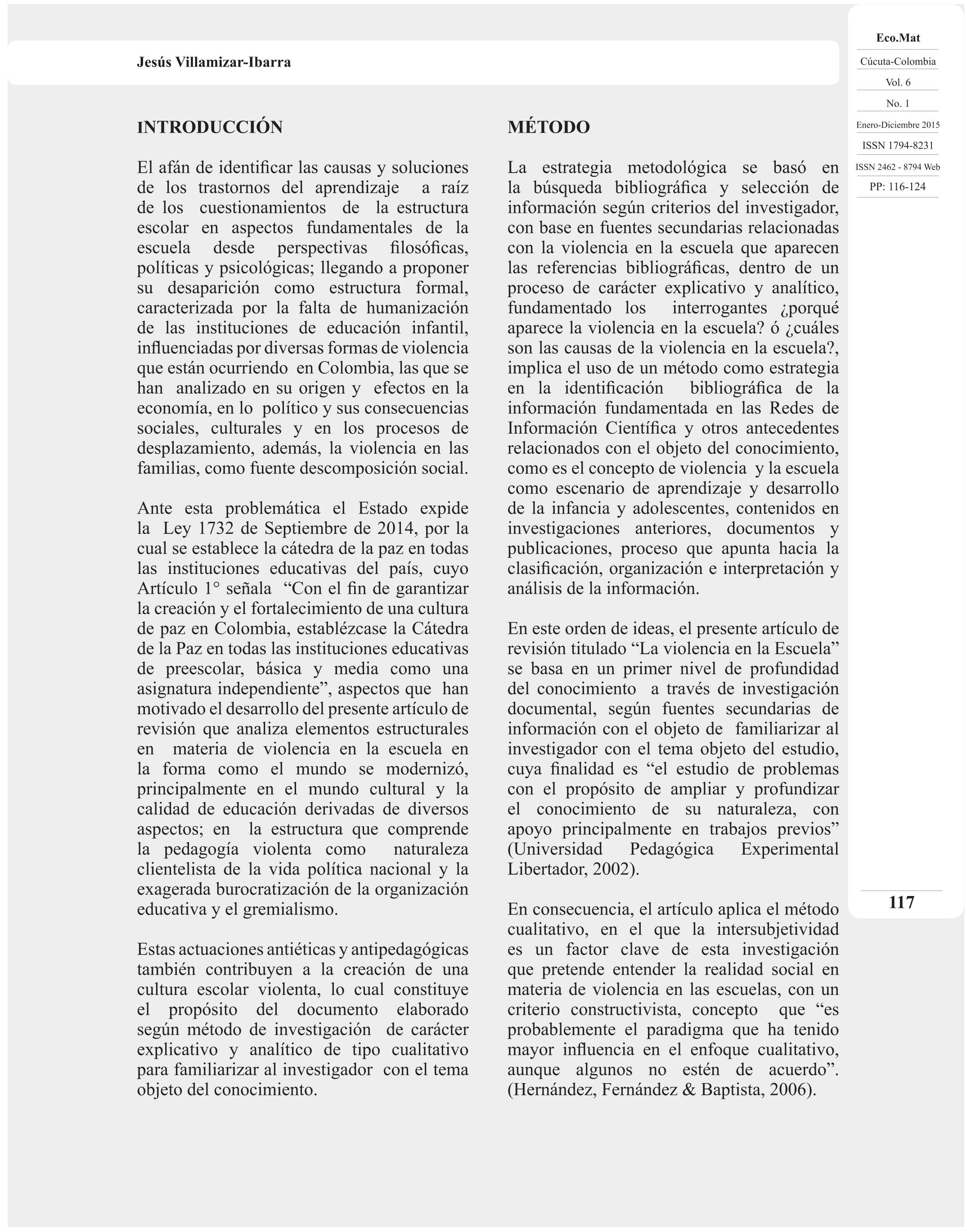




\section{LA VIOLENCIA EN LA ESCUELA}

"Uno de los temas que sin menor duda debe preocupar a cualquier sociedad es la educación de su niñez y juventud" (Parra, 1998, p. 11).

A propósito de esta preocupación, se ha desarrollado en el mundo contemporáneo un enorme sistema de prácticas $\mathrm{y}$ conocimientos que abarcan desde las ciencias y administrativas hasta los más avanzados estudios neurológicos que permiten identificar las causas y soluciones de los trastornos del aprendizaje. Es natural que, desde la enorme complejidad del problema educativo, sobreabunden los trabajos e investigaciones sobre el tema (Parra, Gonzales, Moritz, Blandon \& Bustamante, 1998).

Los estudiosos e intelectuales, han puesto el dedo en la llaga cuestionando de raíz la estructura escolar. Illich y Mandel entre otros, han denunciado aspectos fundamentales de la escuela desde perspectivas filosóficas, políticas y psicológicas; llegando a proponer su desaparición como estructura formal. Sin embargo, parece no haber ocurrido nada significativo que apunte a una humanización de las instituciones de educación infantil.

Todo problema educativo en la escuela ha tendido a resolverse por el camino del currículo, de tal manera que la sexualidad, la salud, relaciones interpersonales, la participación democrática, los derechos humanos y la justicia se han convertido rápidamente en asignaturas que ocupan todos los tiempos de la jornada escolar, sin dejar lugar al juego libre, a la interacción espontánea y voluntaria o al ocio creativo.

La violencia en Colombia ha sido estudiada desde varios aspectos. Se ha analizado su origen y los efectos en la economía, carácter político y sus consecuencias sociales, culturales y en los procesos de desplazamiento.

Se han realizado estudios de la violencia en las familias, como contribuyentes en la descomposición social, pero hasta ahora se ha empezado a investigar el papel que juega

\section{La violencia en la escuela}

la escuela en la violencia colombiana. Vale la pena preguntarnos: ¿"cumple la escuela colombiana actual con su papel de ser templo del saber y de la formación de ciudadanos para la democracia y la paz"? ¿Ofrece una cultura alternativa que venga a contrarrestar, desde la competencias ciudadanas, la cultura de la violencia que vive todo el territorio colombiano? ¿Qué formas de violencia se conocen? ¿Cuáles son las manifestaciones más importantes? ¿Cuál es el papel que desempeñan los maestros, los alumnos, los padres de familia? ¿Qué relación tiene la violencia nacional con la violencia escolar? ¿Qué impacto tiene dentro de la pedagogía? ¿Puede hablarse de una pedagogía violenta, de una escuela violenta?

En este sentido, el Estado Colombiano está realizando esfuerzos significativos en materia de paz, es así que aparece la Ley 1732 de Septiembre de 2014, por la cual se establece la cátedra de la paz en todas las instituciones educativas del país, cuyo Artículo $1^{\circ}$ señala "Con el fin de garantizar la creación y el fortalecimiento de una cultura de paz en Colombia, establézcase la Cátedra de la Paz en todas las instituciones educativas de preescolar, básica y media como una asignatura independiente".

A la escuela se le han pedido y se le han adjudicado misiones diversas y en algunos casos contradictorias, durante mucho tiempo. Motor de desarrollo, de modernizaciones económicas y tecnológicas en relación con el trabajo, canal de movilización social, como mecanismo de reproducción burguesa, como vía para acabar con los males del hombre en el mundo de hoy. Pero muy poco desde su propia realidad, desde lo que sucede dentro de las aulas.

La escuela se ha mirado como una institución donde se lleva a cabo procesos de creación y distribución de conocimientos. Pero esta no debe olvidar su papel de formadora de ciudadanos. En ella se viven hechos que son un aprendizaje de la vida en sociedad. Todo esto es necesario para la formación de ciudadanos, miembros de una sociedad democrática. 


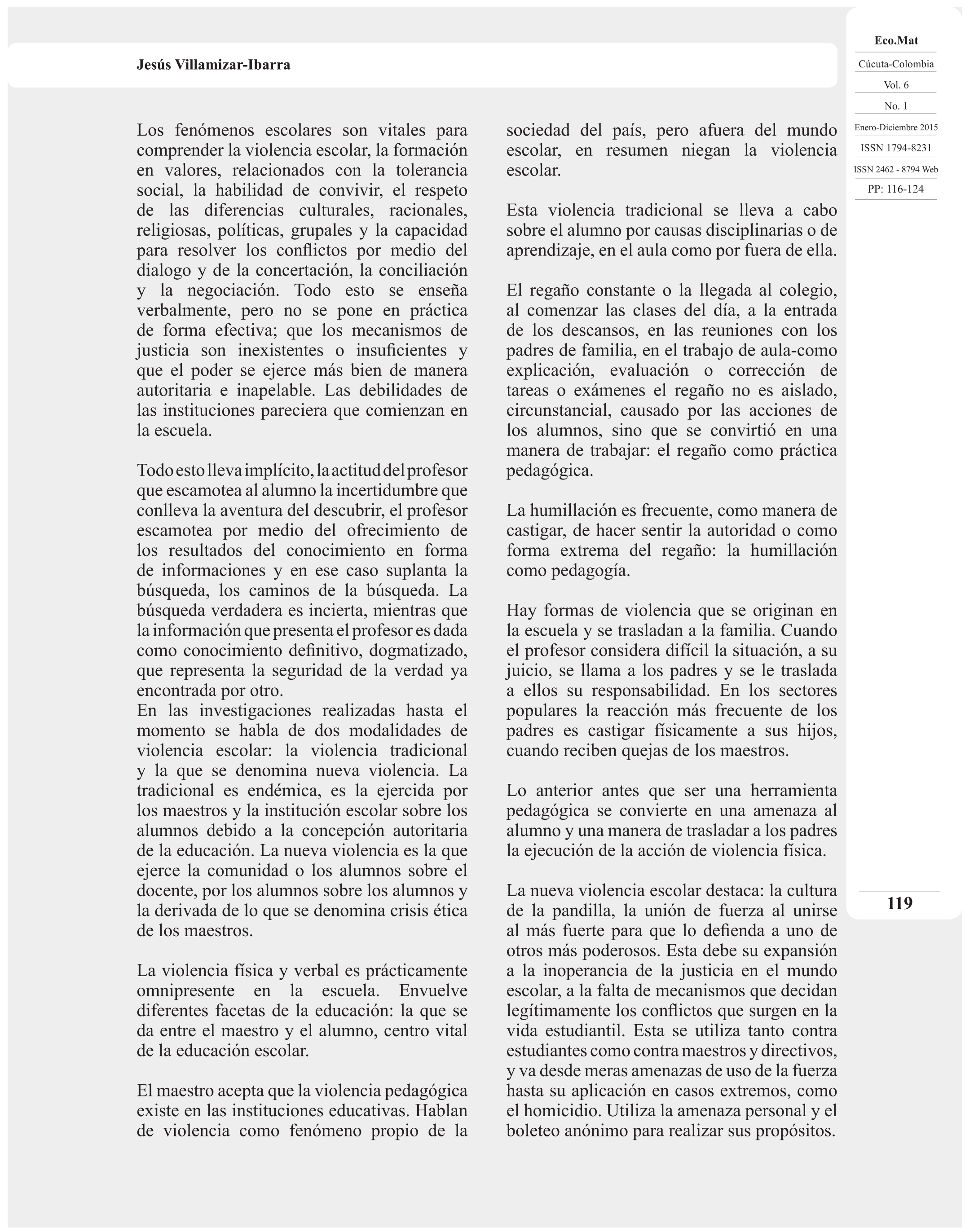


Eco.Mat

Cúcuta-Colombia

Vol. 6

No. 1

Enero-Diciembre 2015

ISSN 1794-8231

ISSN 2462 - $8794 \mathrm{We}$

PP: 116-124
Creación de espacios de nadie a donde no llega la autoridad de maestros y directivos, incluso con la anuencia y, a veces con el beneplácito de los docentes. Estos espacios construyen su propia cultura en que es válida la violencia física como forma natural de interacción.

La degradación de la ética docente crea situaciones que dificultan la formación de una ética escolar, la formación de los alumnos como ciudadanos y generan violencia personal e institucional entre los docentes y con los alumnos.

Es muy común hablar de la cultura del menor esfuerzo en el trabajo escolar de los docentes, hacen difícil la labor de los nuevos maestros que llegan con ideas y deseos de innovar y de trabajar eficazmente. Lleva a conflictos entre el cuerpo profesional y al abandono del trabajo docente, el trabajo del docente ya no es una forma de vida sino que se transforma en una manera burocrática de supervivencia.

Es importante aclarar algunas de las raíces, sus orígenes y el marco socio-educativo en que opera la pedagogía violenta.

Aparecen los siguientes elementos estructurales que la hacen posible: A) La forma como el mundo se modernizó, principalmente en el mundo cultural y la calidad de educación derivadas de los aspectos:

1. La expansión del mundo escolar que se llevó a cabo en poco tiempo. La improvisación en la formación de maestros. La democratización del ingreso 120 a la escuela, pero no hacia el mejoramiento de la calidad de la educación.

2. Con base a que la educación es el motor del desarrollo porque da conocimiento y éste impulsa la productividad se crean planes de formación de maestros que privilegian la transmisión de conocimiento sobre la creación de conocimiento. Los maestros fueron especializados en distribuir y no en crear conocimiento. Esto llevo a que

\section{La violencia en la escuela}

los maestros tengan que ser autoritarios, memoristas aburridores y por lo tanto a dar importancia en la cultura escolar al control, la disciplina y el castigo.

3. Se exaltó la importancia del currículo y se ha difundido la idea de que educar es fundamentalmente aplicar un currículo. Olvidando la vida escolar, la manera como se vive la organización social, el poder, la autoridad, la justicia, las relaciones entre maestros y alumnos y la forma como se concibe el conocimiento educando al ciudadano.

La pedagogía de la vida escolar se olvidó y se hizo énfasis en la pedagogía de la distribución curricular.

B) Otro elemento de la estructura que comprende la pedagogía violenta es la naturaleza clientelista de la vida política nacional. El clientelismo se preocupó solamente de la expansión escolar y del lleno de las escuelas con maestros sin preparación, sin vocación académica y con una visión de burócrata. Este despojo de la calidad es una forma de violencia, que se ejerce en la juventud, se da a más personas algo que cada vez sirve menos.

C) La exagerada burocratización de la organización educativa y el gremialismo mal entendido que defiende las actuaciones antiéticas y antipedagógicas que también contribuye a la creación de una cultura escolar violenta.

"Es entendida la violencia escolar como la acción $\mathrm{u}$ omisión intencionalmente dañina ejercida entre los miembros de la comunidad educativa (alumnos, profesores, padres, personal subalterno) y que se produce dentro de los espacios físicos que le son propios (instalaciones escolares), bien en otros espacios directamente relacionados con lo escolar (alrededores de la escuela o lugares donde se desarrollan actividades extraescolares" (Díaz, 2005, p. 37). 
El acoso escolar (también conocido como hostigamiento escolar, matoneo escolar o por su término en ingles Bullying) es cualquier forma de maltrato psicológico, verbal o físico producido entre escolares de forma reiterada a lo largo de un tiempo determinado. Estadísticamente, el tipo de violencia dominante es el emocional y se da mayoritariamente en el aula y patio de los centros escolares. Los protagonistas de los casos de acoso escolar suelen ser niños y niñas en proceso de entrada en la adolescencia (12-13 años), siendo ligeramente mayor el porcentaje de niñas en el perfil de víctimas. El acoso escolar es una especie de tortura, metódica y sistemática, en la que el agresor sume a la víctima, a menudo con el silencio, la indiferencia o la complicidad de otros compañeros (Fernández, 2014, p.2).

"El 43,6\% de todos los encuestados (as) admitió que alguna vez ha agredido a un compañero (a), ridiculizándolo, golpeándolo, excluyéndolo o con amenazas. El 16,4\% están solos en el momento de la agresión. El $34 \%$ sentimiento posterior del agresor: $32,5 \%$ satisfechos, $30,9 \%$ no identifican algún sentimiento. E1 51,4\% de todos los encuestados (as) dicen haber sufrido agresiones. El 58,7\% de las agresiones ocurren en el salón de clase y el $18 \%$ en el patio de recreo" (Universidad Javeriana, 2006).

La encuesta Nacional de deserción escolar ENDE 2009 (citado por Román y Murillo, 2011) estableció que el $13 \%$ es por maltrato de compañeros, $15 \%$ conflicto y violencia, $13 \%$ maltrato docentes y directivos. Estos resultados indican que el tipo de violencia dominante es el emocional y se da principalmente en el aula y en el patio de los centros escolares. Los involucrados en los casos de acoso suelen ser niños y niñas en proceso de entrada en la adolescencia (12-13 años) siendo ligeramente mayor el porcentaje de niñas en el perfil de víctimas. (Román y Murillo, 2011).

"El estudio Revista CEPAL de la comisión económica para américa latina 2011 estableció que el $51,1 \%$ de los estudiantes de sexto grado de educación primaria de los 16 países latinoamericanos examinados dicen haber sido víctimas de insultos, amenazas, golpes o robos (Bullying) por parte de sus compañeros de escuela. La agresión más frecuente fue el robo $(39,4 \%)$, seguida de la violencia verbal (26,6\%) y la violencia física (16,5\%)" (Román y Murillo, 2011).

Tabla I. CARACTERÍSTICAS DE LA VÍCTIMA Y VICTIMARIO

\begin{tabular}{|c|c|}
\hline \multicolumn{2}{|c|}{ VICTIMA O VICTIMARIO } \\
\hline VICTIMA & VICTIMARIO \\
\hline Baja autoestima & Permisibilidad excesiva \\
\hline Persona sola y de pocos amigos & Impulsividad \\
\hline Pocas habilidades comunicativas y sociales & Conducta violenta \\
\hline $\begin{array}{l}\text { Tiene alguna característica que lo distingue } \\
\text { de los demás (usa gafas, es muy alto, muy } \\
\text { bajo, es muy gordo o flaco). }\end{array}$ & Dificultad para relacionarse \\
\hline Timidez & Dominación \\
\hline & Intolerancia \\
\hline & Justifican la violencia \\
\hline & Arrogancia \\
\hline & Usa insultos o apodos despectivos \\
\hline & $\begin{array}{l}\text { Ausencia de empatía (incapacidad para } \\
\text { ponerse en el lugar del otro- insensible al } \\
\text { sufrimiento de los otros). }\end{array}$ \\
\hline
\end{tabular}


Eco.Mat

Cúcuta-Colombia

Vol. 6

No. 1

Enero-Diciembre 2015 ISSN 1794-8231

ISSN 2462 - $8794 \mathrm{Web}$

PP: $116-124$

Acosador-víctima no tienen que conocerse. El acoso es de 24 horas, hay persistencia total, en el 2009 las denuncias por ciber-matoneo crecieron en un $300 \%$, montaje (videosfotos), (Diario El Tiempo, 2014)

Caso de suicidio por Bullying. El adolescente de Búfalo, Rodemeyer se suicidó durante el mes de septiembre de 2011. El chico de 14 años, quien hizo un video para el proyecto de anuncios de servicio público IT GETS BETTER, soportó un intenso hostigamiento, debido a su sexualidad, por parte de sus compañeros en la Preparatorio Williansville North. En su video de IT GETS BETTER, Rodemeyer citó a Lady Gaga como una inspiración debido a su mensaje de aceptación, el miércoles Gaga utilizo Twitter para abogar

\section{La violencia en la escuela}

por la creación de leyes contra el acoso escolar.

Gaga mencionó que "Los últimos días los he pasado reflexionando, llorando y gritando. Tengo tanta ira" (CNN México, 2011, p.1). "Es difícil sentir amor cuando la crueldad toma la vida de alguien...el Bullying debe ser ilegal. Es un crimen de odio, me reuniré con nuestro presidente. No voy a dejar de luchar. Esto tiene que terminar. Nuestra generación tiene el poder de terminar con él". (CNN México, 2011, p.1). El suicidio del adolescente acosado está impulsando a la cantante a abogar por penas contra el acoso escolar. (CNN México, 2011).

Acoso escolar o Bullying, humillación, suicidio, conducta negativa, ridiculización, maltrato físico y verbal, intimidación, aislamiento, violencia escolar.

En Colombia los fundamentos constitucionales

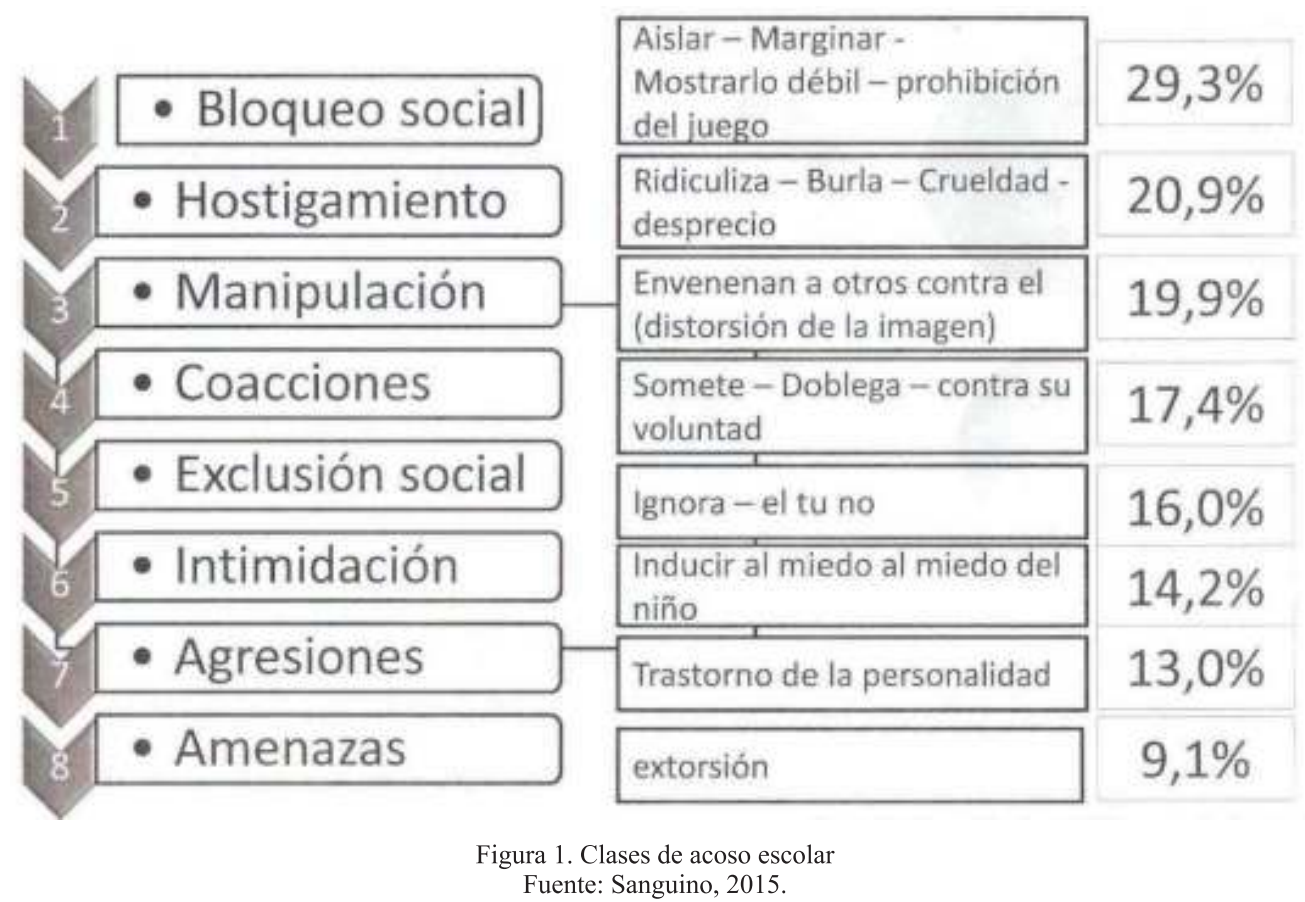

y legales se contemplan en los artículos 41 43-45, 95 de la Carta Magna y los desarrollos normativos de la educación en la Ley 115/94, Decreto 1860 de 1994 y la Ley 715 de 2001, además, el Código de la infancia y la adolescencia y la nueva ley de convivencia escolar Ley 1620 del 15 de Marzo de 2013. Es el objetivo de la ley, en concordancia con el mandato constitucional y la Ley General de Educación, Ley 115 de 1994, proponer $\mathrm{y}$ fortalecer la formación ciudadana y el ejercicio de los derechos humanos, sexuales 


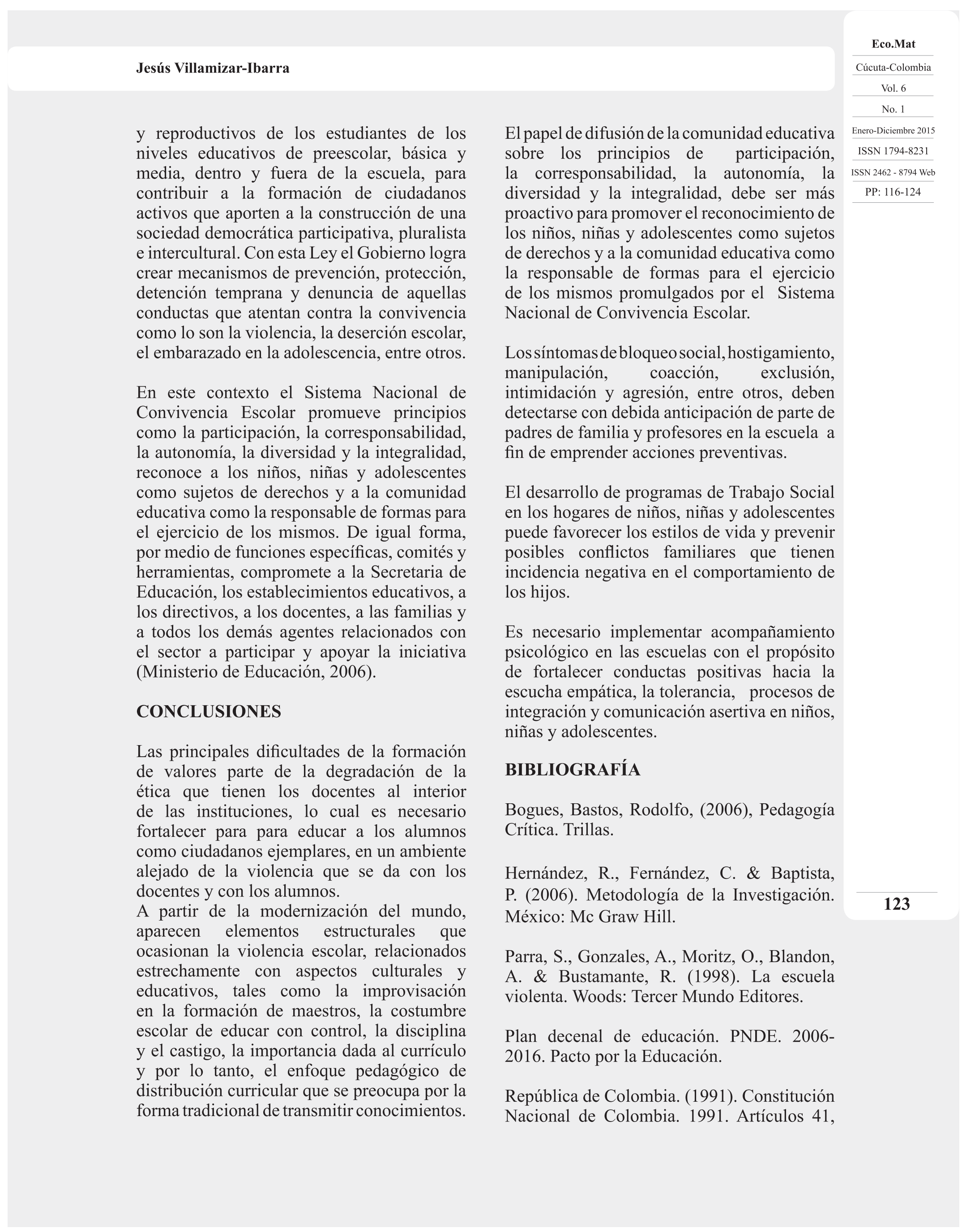


Eco.Mat

Cúcuta-Colombia

Vol. 6

No. 1

Enero-Diciembre 2015 ISSN 1794-8231

ISSN $2462-8794 \mathrm{Web}$ PP: 116-124
43-45, 95. Bogotá: Diario Oficial.

República de Colombia. (1994). Decreto 1860/94. Bogotá: Diario Oficial.

República de Colombia. (1994). Ley 115/94 Ley General de Educación Bogotá: Diario Oficial.

República de Colombia. (2001). Ley 715/2001. Bogotá: Diario Oficial.

República de Colombia. (2006). Ley 1098 de 2006, artículos 15 y 39. Bogotá: Diario Oficial.

Universidad Pedagógica Experimental Libertador, (2002), Manual de Trabajo de Grado de especialización y maestría y tesis doctorales, Caracas.

Díaz, M. (2005). Por qué se produce la violencia escolar y como prevenirla. Revista Iberoamericana de Educación.

Fernández, C. El bullying. Recuperado de: http://www.eduinnova.es/dic09/bullyng.pdf

Universidad Javeriana. (2006). Estudio sobre el fenómeno del matoneo o Bullying en Colombia. Bogotá: Unijaveriana.

Ministerio de Educación Nacional. (2009). Encuesta nacional de deserción escolar. Recuperado de: http://www.mineducacion. gov.co/1621/articles293664_archivo_pdf_ resultados_ETC.pdf

124

Román, M. y Murillo, F.J. (2011). América

\section{La violencia en la escuela}

ciber matoneo. Recuperado de: http://www. eltiempo.com/opinion/columnistas/protejasedel-cibermatoneo-guillermo-santos-calderoncolumnista-el-tiempo/14746818

CNN México. (2011). Lady Gaga: "El 'bullying' es un crimen de odio, debe ser ilegal". Recuperado de: http://mexico.cnn. com/entretenimiento/2011/09/26/gaga-elbullying-es-un-crimen-de-odio-debe-serilega. Latina: Violencia entre estudiantes y desempeño escolar. Revista CEPAL, 104, pp. 37-54.

Delgado, M. (2009). Comportamientos antisociales en los centros escolares. Recuperado de: http://www.csi-csif.es/ andalucia/modules/mod ense/revista/pdf/ Numero_16/MAGDALEN̄A_DELGADO 1. pdf

Diario El Tiempo. (2014). Protéjase del 